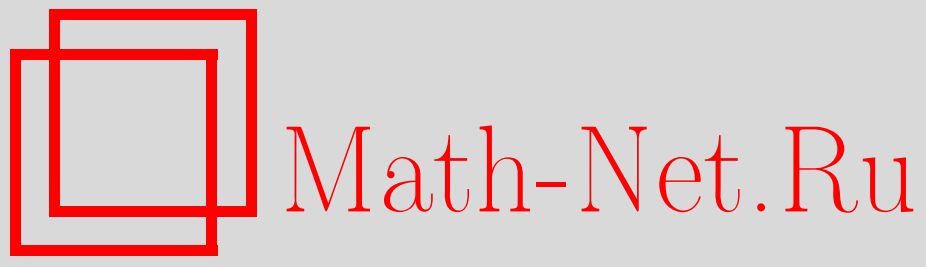

А. А. Досиев, Гомологические размерности алгебры целых функций от элементов нильпотентной алгебры Ли, Функи. анализ и его прил., 2003, том 37, выпуск $1,73-77$

DOI: https://doi.org/10.4213/faa137

Использование Общероссийского математического портала MathNet.Ru подразумевает, что вы прочитали и согласны с пользовательским соглашением

http://www . mathnet.ru/rus/agreement

Параметры загрузки:

IP : 18.208 .226 .222

26 апреля 2023 г., 17:58:51

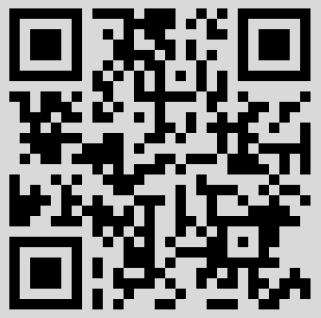




\title{
Гомологические размерности алгебры целых функций от элементов нильпотентной алгебры Ли
}

\author{
(c) 2003. А. А. ДОсиЕВ
}

Данная статья посвящена гомологическим характеристикам оболочки АренсаМайкла (см. [1, гл. 5, п. 2.21]) $\mathscr{O}_{\mathfrak{g}}$ универсальной обертывающей алгебры $\mathscr{U}(\mathfrak{g})$ конечномерной нильпотентной алгебры Ли $\mathfrak{g}$. Алгебра Фреше $\mathscr{O}_{\mathfrak{g}}$ интерпретируется как алгебра целых функций от элементов алгебры Ли g (см. [2]). Некоммутативные версии алгебры голоморфных функций от элементов произвольной алгебры Ли на полидиске рассмотрены в $[2,3]$. Если $D-$ полидиск в $\mathbb{C}^{n}(n=\operatorname{dim}(\mathfrak{g}))$, то алгебра $\mathscr{O}_{\mathfrak{g}}(D)$ голоморфных функций от элементов алгебры Ли $\mathfrak{g}$ на полидиске $D$ определялась как некоторое (см. [2]) мультинормированное пополнение алгебры $\mathscr{U}(\mathfrak{g})$. Если $D=\mathbb{C}^{n}$, то $\mathscr{O}_{\mathfrak{g}}(D)$ сводится к оболочке Аренса-Майкла $\mathscr{O}_{\mathfrak{g}}$. Пусть $e=\left(e_{1}, \ldots, e_{n}\right)$ - базис в нильпотентной алгебре Ли $\mathfrak{g}$, согласованный с нижним центральным рядом $\mathfrak{g}^{(s)}(s \geqslant 1)$ этой алгебры, т.е. присоединенное представление алгебры Ли $\mathfrak{g}$ приводится к строго треугольному виду относительно этого базиса. Такой базис мы назовем треугольным базисом в $\mathfrak{g}$. Каждому элементу $f \in \mathscr{O}_{\mathfrak{g}}(D)$ можно сопоставить единственным способом формальный степенной ряд $\sum_{J} a_{J} e^{J}$, где $J=\left(j_{1}, \ldots, j_{n}\right)-$ мультииндекс и $e^{J}=e_{1}^{j_{1}} \ldots e_{n}^{j_{n}}$ (см. [3]). Мы говорим, что множество $\left\{e^{J}\right\}$ является однородным базисом в $\mathscr{O}_{\mathfrak{g}}(D)$, если для каждого $f$ указанный ряд однородно сходится к $f$, т. е. $f$ может быть представлен в виде абсолютно сходящегося ряда $\sum_{k} f_{k}$, где $f_{k}=\sum_{|J|=k} a_{J} e^{J},|J|=\sum_{i=1}^{n} p_{i} j_{i}, p_{i}=\max \left\{s: e_{i} \in \mathfrak{g}^{(s)}\right\}$. Если любой $f \in \mathscr{O}_{\mathfrak{g}}(D)$ представляется в виде безусловно (соответственно абсолютно) сходящегося степенного ряда $\sum_{J} a_{J} e^{J}$, то мы говорим, что множество $\left\{e^{J}\right\}$ является безусловным (соответственно абсолютным) базисом в $\mathscr{O}_{\mathfrak{g}}(D)$. В [3] доказано существование однородного базиса в алгебре $\mathscr{O}_{\mathfrak{g}}(D)$ для нильпотентной алгебры Ли $\mathfrak{g}$, допускающей положительную целочисленную градуировку подпространств.

С целью вычисления гомологических характеристик алгебр $\mathscr{O}_{\mathfrak{g}}(D)$ мы рассмотрим, как и в чисто алгебраическом случае (см. [4, гл. 13]), вопросы существования свободной резольвенты Кошуля для тривиального модуля над пополнениями алгебры $\mathscr{U}(\mathfrak{g})$ и бимодульной резольвенты Кошуля этих алгебр. Мы покажем, что если для некоторого треугольного базиса $e$ алгебры Ли $\mathfrak{g}$ множество упорядоченных мономов $\left\{e^{J}\right\}$ является делимым (см. ниже) базисом в некотором локально выпуклом пополнении $\mathscr{A}$ алгебры $\mathscr{U}(\mathfrak{g})$, то комплекс Кошуля, порожденный тривиальным $\mathscr{A}$-модулем, допустим (т. е. $\mathbb{C}$-расщепим). Кроме того, если $\mathfrak{g}^{(3)}=[\mathfrak{g},[\mathfrak{g}, g]]=\{0\}$, то алгебра $\mathscr{A}=\mathscr{O}_{\mathfrak{g}}$ имеет $\mathscr{O}_{\mathfrak{g}}$-бимодульную резольвенту Кошуля. Таким образом, естественное вложение $\mathscr{U}(\mathfrak{g}) \rightarrow \mathscr{O}_{\mathfrak{g}}$ является абсолютной локализацией в смысле Дж. Тейлора [5], если ступень нильпотентности алгебры Ли $\mathfrak{g}$ равна двум.

Комплекс Кошуля. Категория всех комплексных хаусдорфовых полных локально выпуклых пространств (соответственно алгебр с единицей и с совместно непрерывной операцией умножения) обозначается через LCS (соответственно 
LCA). Пусть $\mathscr{A} \in \mathbf{L C A}$ и $\mathfrak{X}-$ левый локально выпуклый $\mathscr{A}$-модуль, т.е. $\mathfrak{X} \in \mathbf{L C S}$ и отображение внешнего умножения $\mathscr{A} \times \mathfrak{X} \rightarrow \mathfrak{X}$ совместно непрерывно. Категория всех левых (соответственно правых, двусторонних) $\mathscr{A}$-модулей обозначается через $\mathscr{A}$-mod $($ соответственно $\bmod -\mathscr{A}, \mathscr{A}$-mod- $\mathscr{A})$. Проективной гомологической размерностью $\mathrm{dh}_{\mathscr{A}} \mathfrak{X}$ модуля $\mathfrak{X}$ называется длина его самой короткой проективной резольвенты. Гомологическая биразмерность $\mathrm{db} \mathscr{A}$ алгебры $\mathscr{A}$ есть проективная гомологическая размерность алгебры $\mathscr{A}$ как $\mathscr{A}$-бимодуля [6, гл. 3]. Левая проективная глобальная гомологическая размерность dg $\mathscr{A}$ алгебры $\mathscr{A}$ определяется как $\sup \left\{\operatorname{dh}_{\mathscr{A}} \mathfrak{X}: \mathfrak{X} \in \mathscr{A}\right.$-mod $\}$. Левая проективная малая (соответственно средняя) размерность ds $\mathscr{A}$ (соответственно $\mathrm{dm} \mathscr{A})$ есть верхняя грань величин $\mathrm{dh}_{\mathscr{A}} \mathfrak{X}$, взятая по всем конечномерным (соответственно неприводимым) модулям $\mathfrak{X}[6$, гл. 3$]$. Пусть теперь $\mathfrak{X} \in \mathscr{A}$-mod и $\mathfrak{Y} \in \bmod -\mathscr{A}$. Мы используем обозначение $\mathfrak{Y} \perp \mathfrak{X}$ (см. [5] и [6, гл. 3, п. 3.8]), если все производные функторы от функтора тензорного (над $\mathscr{A}$ ) произведения $\mathfrak{Y} \widehat{\otimes} \mathscr{A} ?$, примененные к модулю $\mathfrak{X}$, являются нулевыми.

Пусть $\mathfrak{X} \in \mathbf{L C S}, \mathscr{L}(\mathfrak{X})$ - пространство всех непрерывных линейных операторов, действующих в $\mathfrak{X}$, и пусть $\alpha: \mathfrak{g} \rightarrow \mathscr{L}(\mathfrak{X})$ - представление конечномерной алгебры Ли $\mathfrak{g}$ в пространстве $\mathfrak{X}$, т. е. $\mathfrak{X}$ есть $\mathfrak{g}$-модуль. Определим образ $\operatorname{Im}(\alpha)$ представления $\alpha$ как алгебраическую сумму $\sum_{u \in \mathfrak{g}} \operatorname{im}(\alpha(u))$. Если $I-$ идеал алгебры Ли $\mathfrak{g}$, то нетрудно показать, что $\operatorname{Im}\left(\left.\alpha\right|_{I}\right)$, где $\left.\alpha\right|_{I}-$ сужение представления $\alpha$ на $I$, есть $\mathfrak{g}$-подмодуль в $\mathfrak{X}$. Тогда $\alpha$ индуцирует факторпредставление $\alpha_{I}: \mathfrak{g} / I \rightarrow \mathscr{L}\left(\mathfrak{X} / \operatorname{Im}\left(\left.\alpha\right|_{I}\right)\right), \alpha_{I}\left(u^{\sim}\right)=\alpha(u)^{\sim}$, где $u^{\sim}-$ класс элемента $u(\bmod I)$, а $\alpha(u)^{\sim}$ - оператор в факторпространстве $\mathfrak{X} / \operatorname{Im}\left(\left.\alpha\right|_{I}\right)$, индуцированный оператором $\alpha(u)$.

Пусть $\mathfrak{g}-$ нильпотентная алгебра Ли с треугольным базисом $e=\left(e_{1}, \ldots, e_{n}\right)$. Положим $l_{i}=e_{n-i+1}, T_{i} \in \mathscr{L}(\mathfrak{X}), T_{i} x=\alpha\left(l_{i}\right) x, 1 \leqslant i \leqslant n$, и пусть $D_{k}=$ $\sum_{i=1}^{k} \operatorname{im}\left(T_{i}\right)$. Ясно, что подпространство $I_{k}$, порожденное элементами $l_{1}, \ldots, l_{k}$, является идеалом в $\mathfrak{g}$ и $D_{k}=\operatorname{Im}\left(\left.\alpha\right|_{I_{k}}\right)$. В частности, $\operatorname{Im}(\alpha)=D_{n}$. Пусть $\mathfrak{X}_{-1}=\mathfrak{X} / D_{n}$, и пусть $\varepsilon: \mathfrak{X} \rightarrow \mathfrak{X}_{-1}-$ факторотображение. Рассмотрим индуцированные операторы $\bar{T}_{i}=\alpha_{I_{k}}\left(l_{i}^{\sim}\right) \in \mathscr{L}\left(\mathfrak{X} / D_{k}\right), i \geqslant k+1$, и цепной комплекс Кошуля $\operatorname{Kos}(\mathfrak{X}, \alpha): 0 \longleftarrow \mathfrak{X} \stackrel{d_{0}}{\longleftarrow} \mathfrak{X} \otimes \mathfrak{g} \longleftarrow d_{1} \ldots \stackrel{d_{m-1}}{\longleftarrow} \mathfrak{X} \otimes \wedge^{m} \mathfrak{g} \stackrel{d_{m}}{\longleftarrow} \cdots$ с дифференциалом $d_{m}(x \otimes \underline{u})=\sum_{i=1}^{m}(-1)^{i} \alpha\left(u_{i}\right) x \otimes \underline{u}_{i}+\sum_{i<j}(-1)^{i+j-1} x \otimes\left[u_{i}, u_{j}\right] \wedge \underline{u}_{i j}$, где $\underline{u}=u_{1} \wedge \ldots \wedge u_{m+1} \in \wedge^{m+1} \mathfrak{g}$, а $\underline{u}_{i}$ (соответственно $\left.\underline{u}_{i j}\right)-$ вектор из пространства $\wedge^{m} \mathfrak{g}$ (соответственно $\left.\wedge^{m-1} \mathfrak{g}\right)$, полученный из $\underline{u}$ путем удаления вектора $u_{i}$ (соответственно векторов $u_{i}, u_{j}$ ). Пополненный комплекс Кошуля $0 \leftarrow \mathfrak{X}_{-1} \stackrel{\varepsilon}{\longleftarrow} \operatorname{Kos}(\mathfrak{X}, \alpha)$ с пополняющим отображением $\varepsilon$ обозначим через $\operatorname{Kos}^{+}(\mathfrak{X}, \alpha)$.

Лемма 1. Пусть все $\bar{T}_{k+1} \in \mathscr{L}\left(\mathfrak{X} / D_{k}\right)$ обладают непрерывными левыми обратными операторами. Тогда комплекс $\operatorname{Kos}^{+}(\mathfrak{X}, \alpha)$ допустим.

Отмеченные пополнения. Пусть $\mathfrak{g}-$ конечномерная алгебра Ли, $\Delta(\mathfrak{g})=$ $\left\{\lambda \in \mathfrak{g}^{*}: \lambda\left(\mathfrak{g}^{(2)}\right)=\{0\}\right\}-$ пространство всех лиевых характеров алгебры Ли $\mathfrak{g}$, и пусть $\varepsilon_{\mathfrak{g}}: \mathscr{U}(\mathfrak{g}) \rightarrow \mathbb{C}-$ канонический характер (мультипликативный линейный функционал) алгебры $\mathscr{U}(\mathfrak{g})$, продолжающий тривиальный характер алгебры Ли $\mathfrak{g}$. Хорошо известно $[4$, гл. 13$]$, что противоположная алгебра $\mathscr{U}(\mathfrak{g})^{\text {op }}$ отождествляется с обертывающей алгеброй $\mathscr{U}\left(\mathfrak{g}^{\mathrm{op}}\right)$ противоположной алгебры Ли $\mathfrak{g}^{\mathrm{op}}$. 
Кроме того, между алгебрами $\mathscr{U}(\mathfrak{g})$ и $\mathscr{U}(\mathfrak{g})^{\text {op }}$ существует изоморфизм, который задается с помощью главного антиавтоморфизма $\kappa_{\mathfrak{g}}: \mathscr{U}(\mathfrak{g}) \rightarrow \mathscr{U}(\mathfrak{g}), \kappa_{\mathfrak{g}}(x)=-x$, $x \in \mathfrak{g}$. Рассмотрим теперь алгебру $\mathscr{A} \in \mathbf{L C A}$, которая является пополнением алгебры $\mathscr{U}(\mathfrak{g})$. Мы скажем, что $\mathscr{A}$ является отмеченным пополнением обертывающей алгебры $\mathscr{U}(\mathfrak{g})$, если канонический характер $\varepsilon_{\mathfrak{g}}$ и главный антиавтоморфизм $\kappa_{\mathfrak{g}}$ непрерывны относительно индуцированной из $\mathscr{A}$ топологии. Таким образом, если $\mathscr{A}$ есть отмеченное пополнение алгебры $\mathscr{U}(\mathfrak{g})$, то $\varepsilon_{\mathfrak{g}}$ продолжается до непрерывного характера $\varepsilon_{\mathfrak{g}}: \mathscr{A} \rightarrow \mathbb{C}$ алгебры $\mathscr{A}$ и $\mathscr{A}=\mathscr{A}^{\text {ор }}$ с точностью до топологического изоморфизма алгебр, продолжающего $\kappa_{\mathfrak{g}}$. При этом одномерное пространство $\mathbb{C}$ наделяется структурой $\mathscr{A}$-бимодуля посредством характера $\varepsilon_{\mathfrak{g}}$, и этот бимодуль называется тривиальным модулем и обозначается через $\mathbb{C}\left(\varepsilon_{\mathfrak{g}}\right)$. Мы также пишем $f(0)$ вместо $\varepsilon_{\mathfrak{g}}(f)$ для любого $f \in \mathscr{A}$. Нетрудно показать, что для произвольной конечномерной алгебры Ли $\mathfrak{g}$ все алгебры $\mathscr{O}_{\mathfrak{g}}(D)$ (в частности, $\left.\mathscr{O}_{\mathfrak{g}}\right)$ являются отмеченными пополнениями алгебры $\mathscr{U}(\mathfrak{g})$. Заметим, что пространство всех непрерывных характеров алгебры $\mathscr{O}_{\mathfrak{g}}$ отождествляется с $\Delta(\mathfrak{g})$, так как $\mathscr{O}_{\mathfrak{g}}$ - оболочка Аренса-Майкла алгебры $\mathscr{U}(\mathfrak{g})$. Если $\lambda \in \Delta(\mathfrak{g})$ и $\mathfrak{g}-\lambda$ - подалгебра Ли в $\mathscr{U}(\mathfrak{g})$, состоящая из элементов вида $u-\lambda(u), u \in \mathfrak{g}$, то $\mathcal{O}_{\mathfrak{g}}=\mathcal{O}_{\mathfrak{g}-\lambda}$ с точностью до топологического изоморфизма алгебр. Поэтому $\mathcal{O}_{\mathfrak{g}}$ - отмеченное пополнение алгебры $\mathscr{U}(\mathfrak{g}-\lambda)$. Продолжение тривиального характера алгебры Ли $\mathfrak{g}-\lambda$ на пополнение $\mathscr{O}_{\mathfrak{g}}$ отождествляется с $\lambda$, а соответствующий тривиальный модуль есть $\mathbb{C}(\lambda)$.

Делимый базис. Пусть $\mathfrak{g}$ - конечномерная алгебра Ли и $e=\left(e_{1}, \ldots, e_{n}\right)-$ базис в $\mathfrak{g}$. Для каждого $i$ рассмотрим оператор $\nabla_{e_{i}} \in \mathscr{L}(\mathscr{U}(\mathfrak{g}))$, действующий по правилу $\nabla_{e_{i}}\left(e_{1}^{j_{1}} \cdots e_{n}^{j_{n}}\right)=\delta_{i j} e_{1}^{j_{1}} \cdots e_{i}^{j_{i}-1} \cdots e_{n}^{j_{n}}$, где $\delta_{i j}=1$ при $j_{i} \geqslant 1$ и $\delta_{i j}=0$ при $j_{i}=0$. Мы назовем $\nabla_{e_{i}}$ операторами деления в $\mathscr{U}(\mathfrak{g})$ относительно базиса $e$. Рассмотрим подпространство $\mathscr{U}_{i}^{e} \subseteq \mathscr{U}(\mathfrak{g})$, состоящее из тех полиномов $\sum_{J} a_{J} e^{J}$, для которых $a_{J}=0$ всякий раз, когда $j_{i+k} \geqslant 1$ для некоторого $k$, $k \geqslant 1$, где $J=\left(j_{1}, \ldots, j_{n}\right), 1 \leqslant i \leqslant n$. Очевидно, что $\mathscr{U}_{1}^{e} \subseteq \mathscr{U}_{2}^{e} \subseteq \cdots \subseteq \mathscr{U}_{n}^{e}=$ $\mathscr{U}(\mathfrak{g})$ и $\mathscr{U}_{i}^{e}$ является инвариантным подпространством относительно оператора деления $\nabla_{e_{i}}$ для каждого $i$.

Пусть теперь $\mathfrak{g}-$ нильпотентная алгебра Ли, $e-$ ее треугольный базис и $\mathscr{A} \in$ LCA - алгебра, которая является пополнением алгебры $\mathscr{U}(\mathfrak{g})$. Множество $\left\{e^{J}\right\}$ всех упорядоченных мономов в $\mathscr{U}(\mathfrak{g})$ называется делимым базисом в $\mathscr{A}$, если оно является однородным базисом в $\mathscr{A}$ и все операторы деления $\nabla_{e_{i}} \in \mathscr{L}\left(\mathscr{U}_{i}^{e}\right)$, $1 \leqslant i \leqslant n$, непрерывны относительно топологии, индуцированной из $\mathscr{A}$.

Проективная малая размерность. Пусть $\mathfrak{g}-$ конечномерная алгебра Ли и $\mathscr{A}$ - отмеченное пополнение алгебры $\mathscr{U}(\mathfrak{g})$. Тогда алгебра $\mathscr{A}$ наделяется структурой $\mathfrak{g}$-модуля (соответственно $\mathfrak{g}^{\text {ор }-м о д у л я) ~ п о с р е д с т в о м ~ л е в о г о ~(с о о т в е т с т в е н н о ~}$ правого) представления $L_{\mathfrak{g}}: \mathfrak{g} \rightarrow \mathscr{L}(\mathscr{A}), L_{\mathfrak{g}}(u) f=u f$ (соответственно $R_{\mathfrak{g}}: \mathfrak{g}^{\text {op }} \rightarrow$ $\left.\mathscr{L}(\mathscr{A}), R_{\mathfrak{g}}(u) f=f u\right), u \in \mathfrak{g}, f \in \mathscr{A}$. Очевидно, что комплекс Кошуля $\operatorname{Kos}\left(\mathscr{A}, L_{\mathfrak{g}}\right)$ $\left(\right.$ соответственно $\left.\operatorname{Kos}\left(\mathscr{A}, R_{\mathfrak{g}}\right)\right)$, порожденный этим модулем, является комплексом

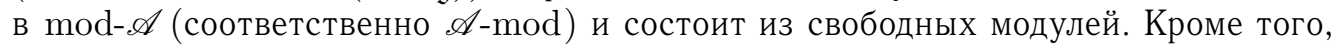
этот комплекс пополняется при помощи непрерывного характера $\varepsilon_{\mathfrak{g}}: \mathscr{A} \rightarrow \mathbb{C}\left(\varepsilon_{\mathfrak{g}}\right)$. Используя лемму 1, можно доказать следующее утверждение.

Теорема 2. Пусть $\mathfrak{g}$ - нильпотентная алгебра Ли u $\mathscr{A}$ - отмеченное пополнение алгебры $\mathscr{U}(\mathfrak{g})$ с делимым базисом. Тогда комплекс Кошуля $\operatorname{Kos}\left(\mathscr{A}, L_{\mathfrak{g}}\right)$ 
(соответственно $\operatorname{Kos}\left(\mathscr{A}, R_{\mathfrak{g}}\right)$ ) является свободной резольвентой правого (соответственно левого) $\mathscr{A}$-модуля $\mathbb{C}\left(\varepsilon_{\mathfrak{g}}\right)$. В частности, $\mathrm{ds}(\mathscr{A})=\mathrm{ds}\left(\mathscr{A}^{\mathrm{op}}\right) \geqslant$ $\operatorname{dim}(\mathfrak{g})$.

Спектр Тейлора. Напомним (см. [7-9]), что спектр Тейлора $\sigma(\alpha)$ представления $\alpha$ конечномерной алгебры Ли $\mathfrak{g}$ на банаховом пространстве $\mathfrak{X}$ (в этом случае $\mathfrak{X} \in \mathscr{O}_{\mathfrak{g}}$-mod) определяется как множество тех $\lambda \in \Delta(\mathfrak{g})$, для которых комплекс Кошуля $\operatorname{Kos}(\mathfrak{X}, \alpha-\lambda)$ не точен. Из теоремы 2 мы получаем следующее утверждение.

Теорема 3. Пусть $\mathfrak{g}-$ нильпотентная алгебра Ли, $\mathscr{A}-$ отмеченное пополнение алгебры $\mathscr{U}(\mathfrak{g})$ с делимым базисом и $\alpha: \mathscr{A} \rightarrow \mathscr{L}(\mathfrak{X})$ - непрерывное представление алгебры $\mathscr{A}$ в банаховом пространстве $\mathfrak{X}$. Тогда $0 \notin \sigma\left(\left.\alpha\right|_{\mathfrak{g}}\right)$ в том и только в том случае, когда $\mathbb{C}\left(\varepsilon_{\mathfrak{g}}\right) \perp \mathfrak{X}$.

Нильпотентная алгебра Ли ступени 2. Пусть $\mathfrak{g}^{(3)}=\{0\}$ и $e=\left(e_{1}, \ldots, e_{n}\right)-$ треугольный базис в $\mathfrak{g}$, такой, что $e_{m+1}, \ldots, e_{n}$ является базисом в $\mathfrak{g}^{(2)}$. Мы скажем, что е является существенным базисом в $\mathfrak{g}$, если для каждого $j, j \geqslant m+1$, существует пара $(i, k), 1 \leqslant i, k \leqslant m$, такая, что $\left[e_{i}, e_{k}\right]=c_{j} e_{j}$ для некоторого ненулевого $c_{j} \in \mathbb{C}$. Очевидно, что такие базисы существуют в произвольной нильпотентной алгебре Ли ступени 2.

Теорема 4. Пусть $\mathfrak{g}-$ конечномерная нильпотентная алгебра Ли ступени 2 и е - ее существенный базис. Тогда множество всех упорядоченных мономов $\left\{e^{J}\right\}$ является абсолютным (и, в частности, безусловным ) базисом в алгебре $\mathscr{O}_{\mathfrak{g}}$.

Следствие 5. Пусть $e=\left(e_{1}, \ldots, e_{n}\right)$ - существенный базис в нильпотентной алгебре Ли $\mathfrak{g}$ ступени 2 , и пусть $\partial_{e_{i}} \in \mathscr{L}(\mathscr{U}(\mathfrak{g})), \partial_{e_{i}}\left(e_{1}^{j_{1}} \cdots e_{n}^{j_{n}}\right)=$ $j_{i} e_{1}^{j_{1}} \cdots e_{i}^{j_{i}-1} \cdots e_{n}^{j_{n}}$, - операторы частных производных. Тогда эти операторы допускают непрерывные продолжения $\partial_{e_{i}} \in \mathscr{L}\left(\mathscr{O}_{\mathfrak{g}}\right), 1 \leqslant i \leqslant n$. В частности, если $f \in \mathscr{O}_{\mathfrak{g}}$ и $f=\sum_{J} a_{J} e^{J}-$ разложение элемента $f$ в $\mathscr{O}_{\mathfrak{g}}$ в абсолютно сходящийся степенной ряд, то для коэффищиентов этого ряда справедлива классическая формула Тейлора $a_{J}=(J !)^{-1}\left(\partial_{e}^{J} f\right)(0)$, где $\partial_{e}^{J}=\partial_{e_{1}}^{j_{1}} \cdots \partial_{e_{n}}^{j_{n}}$, $J=\left(j_{1}, \ldots, j_{n}\right)$.

Пусть теперь $\mathfrak{g}-$ конечномерная алгебра Ли и $d: \mathscr{O}_{\mathfrak{g}} \rightarrow \mathscr{O}_{\mathfrak{g} \times \mathfrak{g}}, d(u)=(u, u)$, $u \in \mathfrak{g},-$ непрерывный гомоморфизм алгебр (диагональное отображение). Пространство $\mathscr{O}_{\mathfrak{g} \times g}\left(=\mathscr{O}_{\mathfrak{g}} \widehat{\otimes} \mathscr{O}_{\mathfrak{g}}\right)$ наделяется структурой правого $\mathscr{O}_{\mathfrak{g}}$-модуля посредством гомоморфизма $d$; точнее, $f * h=f d(h), f \in \mathscr{O}_{\mathfrak{g} \times \mathfrak{g}}, h \in \mathscr{O}_{\mathfrak{g}}$.

СледствиЕ 6. Пусть $\mathfrak{g}-$ конечномерная нильпотентная алгебра Ли ступени 2. Тогда правый $\mathscr{O}_{\mathfrak{g}}$-модуль $\mathscr{O}_{\mathfrak{g} \times \mathfrak{g}}$ является свободным $\mathscr{O}_{\mathfrak{g}}$-модулем.

Используя теоремы 3 и 4 , можно доказать следующее утверждение.

Теорема 7. Пусть е- -ущественный базис в нильпотентной алгебре Ли $\mathfrak{g}$ ступени 2. Тогда множество $\left\{e^{J}\right\}$ всех упорядоченных мономов является делимым базисом в отмеченном пополнении $\mathscr{O}_{\mathfrak{g}}$ алгебры $\mathscr{U}(\mathfrak{g})$. В частности, если $\alpha: \mathfrak{g} \rightarrow \mathscr{L}(\mathfrak{X})$ - банахово представление алгебры Ли $\mathfrak{g} u \lambda \in \Delta(\mathfrak{g})$, то $\lambda \notin \sigma(\alpha)$ в том и только в том случае, когда $\mathbb{C}(\lambda) \perp \mathfrak{X}$, где $\mathbb{C}(\lambda)$ тривиальный модуль над $\mathscr{O}_{\mathfrak{g}}$. 
Алгебра Гейзенберга. Пусть $\mathfrak{g}$ - алгебра Гейзенберга, т. е. $\mathfrak{g}-$ нильпотентная алгебра Ли с одномерным коммутантом, $\lambda \in \Delta(\mathfrak{g})$ и $D_{r}(\lambda)-$ полидиск в $\mathbb{C}^{n}$ $(n=\operatorname{dim}(\mathfrak{g}))$ с центром в точке $\lambda$ мультирадиуса $r(r>0)$. Рассмотрим алгебру $\mathscr{O}_{\mathfrak{g}}\left(D_{r}(\lambda)\right)\left(=\mathscr{O}_{\mathfrak{g}-\lambda}\left(D_{r}(0)\right)\right)$ голоморфных функций от элементов алгебры Ли $\mathfrak{g}$ на полидиске $D_{r}(\lambda)$.

ТЕОРемА 8. Пусть $\mathfrak{g}$ - алгебра Гейзенберга и е - ее треугольный базис. Тогда множество $\left\{e^{J}\right\}$ всех упорядоченных мономов является делимым базисом в отмеченном пополнении $\mathscr{O}_{\mathfrak{g}}\left(D_{r}(\lambda)\right)$ универсальной обертывающей алгебры $\mathscr{U}(\mathfrak{g})$.

Из теорем 2, 3 и 8 можно получить такое утверждение.

СледствиЕ 9. Пусть $\alpha: \mathfrak{g} \rightarrow \mathscr{L}(\mathfrak{X})$ - банахово представление алгебрь Гейзенберга $\mathfrak{g} u \lambda \in \Delta(\mathfrak{g})$. Если $\mathscr{O}_{\mathfrak{g}}\left(D_{r}(\lambda)\right) \perp \mathfrak{X}$ для некоторого полидиска $D_{r}(\lambda)$, mo $\lambda \notin \sigma(\alpha)$.

Бимодульная резольвента. Пусть $\mathfrak{g}-$ конечномерная алгебра Ли, $\mathfrak{X} \in$ $\mathscr{O}_{\mathfrak{g}}-\bmod , \mathfrak{Y} \in \bmod -\mathscr{O}_{\mathfrak{g}}$. Пространство $\mathfrak{Y} \widehat{\otimes} \mathfrak{X}$ превращается в $\mathfrak{g}$-модуль посредством представления $\iota_{\mathfrak{X}}^{\mathfrak{Y}}: \mathfrak{g} \rightarrow \mathscr{L}(\mathfrak{Y} \widehat{\otimes} \mathfrak{X}), \iota_{\mathfrak{X}}^{\mathfrak{Y}}(u) y \otimes x=y \otimes(u \cdot x)-(y \cdot u) \otimes x$, $y \in \mathfrak{Y}, x \in \mathfrak{X}$. Если $\mathfrak{X}=\mathfrak{Y}=\mathscr{O}_{\mathfrak{g}}$, то положим $\iota_{\mathfrak{g}}=\iota_{\mathfrak{X}}^{\mathfrak{Y}}$. Рассмотрим в категории $\mathscr{O}_{\mathfrak{g}}$-mod- $\mathscr{O}_{\mathfrak{g}}$ пополненный комплекс Кошуля $\mathrm{Kos}^{+}\left(\mathscr{\mathscr { O }}_{\mathfrak{g}} \widehat{\otimes}_{\mathfrak{Q}}, \iota_{\mathfrak{g}}\right)$ с пополняющим отображением $\pi: \mathscr{O}_{\mathfrak{g}} \widehat{\otimes} \mathscr{O}_{\mathfrak{g}} \rightarrow \mathscr{O}_{\mathfrak{g}}, \pi(f \otimes g)=f g$.

ТЕОрема 10. Пусть $\mathfrak{g}$ - конечномерная нильпотентная алгебра Ли ступени 2. Тогда комплекс $\operatorname{Kos}^{+}\left(\mathscr{O}_{\mathfrak{g}} \widehat{\otimes} \mathscr{O}_{\mathfrak{g}}, \iota_{\mathfrak{g}}\right)$ допустим, а значит, $\operatorname{Kos}\left(\mathscr{O}_{\mathfrak{g}} \widehat{\otimes} \mathscr{O}_{\mathfrak{g}}, \iota_{\mathfrak{g}}\right)$ является $\mathscr{O}_{\mathfrak{g}}$-бимодульной свободной резольвентой алгебры $\mathscr{O}_{\mathfrak{g}}$.

Из теорем 2, 10 и 7 мы получаем следующее утверждение.

СледствиЕ 11. Пусть $\mathfrak{g}-$ конечномерная нильпотентная алгебра Ли ступени 2. Тогда $\operatorname{ds}\left(\mathscr{O}_{\mathfrak{g}}\right)=\operatorname{dm}\left(\mathscr{O}_{\mathfrak{g}}\right)=\operatorname{dg}\left(\mathscr{O}_{\mathfrak{g}}\right)=\operatorname{db}\left(\mathscr{O}_{\mathfrak{g}}\right)=\operatorname{dim}(\mathfrak{g})$.

СЛЕдСТВИЕ 12. Пусть $\mathfrak{g}-$ конечномерная нильпотентная алгебра Ли ступени 2 и $\mathfrak{X} \in \mathscr{O}_{\mathfrak{g}}$ mod, $\mathfrak{Y} \in \bmod -\mathscr{O}_{\mathfrak{g}}$. Тогда $\mathfrak{Y} \perp \mathfrak{X}$ в том и только в том случае, когда комплекс Кошуля $\operatorname{Kos}\left(\mathfrak{Y} \widehat{\otimes} \mathfrak{X}, \iota_{\mathfrak{X}}^{\mathfrak{Y}}\right)$ точен.

Автор глубоко признателен Ю. В. Туровскому за постановку задачи и за ценные советы и А. Я. Хелемскому, А. С. Файнштейну и Д. Белтице за внимание к работе. Автор также благодарит А. Ю. Пирковского за всестороннее обсуждение результатов заметки.

\section{ЛИТЕРАТУРА}

1. Хелемский A. Я. Банаховы и полинормированные алгебры: общая теория, представления, гомологии. Наука, М., 1989. 2. Досиев A. A. Функц. анализ и его прил., 34, вып. 4, 82-84 (2000). 3. Досиев А. А. Записки науч. сем. ПОМИ, 299, 72-121 (2002). 4. Картан A., Әйленберг С. Гомологическая алгебра. ИЛ, М., 1960. 5. Taylor J. L. Adv. in Math., 9, 183-252 (1972). 6. Хелемский A. Я. Гомология в банаховых и топологических алгебрах. Изд-во МГУ, M., 1986. 7. Fainshtein A. S. J. Operator Theory, 29, 3-27 (1993). 8. Досиев A. A. Функц. анализ и его прил., 35, вып. 4, 80-84 (2001). 9. Dosiev A. A. Spectra of infinite parametrized Banach complexes. J. Operator Theory (в печати). 